Int Arch Allergy Immunol 2021;182:388-398

DOI: $10.1159 / 000511612$
Received: February 22, 2020

Accepted: September 16, 2020

Published online: December 16, 2020

\title{
Diagnostic and Therapeutic Value of Hsa_circ_0002594 for T Helper 2-Mediated Allergic Asthma
}

\author{
Zhenli Huang $^{\mathrm{a}}$ Bohua Fu ${ }^{\mathrm{a}} \quad$ Xuefei Qi ${ }^{\mathrm{a}} \quad$ Yuzhu Xu $^{\mathrm{a}} \quad$ Yong Mou $^{\mathrm{a}}$ Min Zhou ${ }^{\mathrm{a}}$ \\ Yong $\mathrm{Cao}^{\mathrm{a}}$ Guorao $\mathrm{Wu}^{\mathrm{a}}$ Jungang $\mathrm{Xie}^{\mathrm{a}}$ Jianping Zhao ${ }^{\mathrm{a}} \mathrm{Yi} \mathrm{Wang}^{\mathrm{a}}$ \\ Weining Xiong ${ }^{\mathrm{b}}$ \\ aDepartment of Respiratory and Critical Care Medicine, Wuhan Clinical Medical Research Center for Chronic Airway \\ Diseases, Key Laboratory of Pulmonary Diseases of Health Ministry, Key Cite of National Clinical Research Center \\ for Respiratory Disease, Tongji Hospital, Tongji Medical College, Huazhong University of Science and Technology, \\ Wuhan, China; bepartment of Respiratory Medicine, Shanghai Ninth People's Hospital Affiliated to Shanghai Jiao \\ Tong University, School of Medicine, Shanghai, China
}

\section{Keywords}

Asthma $\cdot$ CD4 ${ }^{+}$T cells $\cdot$ hsa_circ_0002594 $\cdot$ Th2

\begin{abstract}
Introduction: Circular RNAs (circRNAs) are an endogenous mircoRNA sponge that could act as potential biomarkers for the diagnosis and treatment of diseases. However, the role of circRNAs in asthma is far from clear. Objective: The aim of this study is to assess the diagnostic and therapeutic value of hsa_circ_0002594 for T helper (Th) 2-mediated allergic asthma. Methods: The expression profiles of hsa circ_0002594 in CD4 ${ }^{+}$T cells were revealed by circRNA microarray. Hsa_circ_0002594 expression was confirmed via quantitative real-time PCR (qRT-PCR) in asthmatic patients and healthy subjects. Hsa_circ_0002594 levels were compared between subgroups. The clinical diagnostic abilities and therapeutic response of hsa_circ_0002594 were evaluated. The analyses utilized included a student's $t$ test, non-
\end{abstract}

Edited by: H.-U. Simon, Bern.

karger@karger.com
www.karger.com/iaa

parametric tests, Spearman's rank-order correlation, Fisher's exact test, and the generation of receiver operating characteristic (ROC) curves. Results: Hsa_circ_0002594 was upregulated and positively correlated with fraction of exhaled nitric oxide while negatively correlated with methacholine dose producing a decrease of $20 \%$ from baseline in forced expiratory volume in the first second (PD20) in $\mathrm{CD}^{+} \mathrm{T}$ cells of asthma. Furthermore, hsa_circ_0002594 expression was higher in subgroups with a family history, skin pricking test (SPT)-positive, or Th2-high. The hsa_circ_0002594-high subgroup was more frequently associated with Th2-high biomarker profiles and positive SPT. Hsa_circ_0002594 was decreased after inhaled corticosteroids (ICS) treatment. ROC curve analyses of hsa_circ_0002594 showed high area under the curve values in the presence of ICS or not. Conclusions: Our data suggested that hsa_circ_0002594 was upregulated in $\mathrm{CD} 4^{+} \mathrm{T}$ cells and might have potential value in the diagnosis and treatment of Th2-mediated allergic asthma.

(c) 2020 S. Karger AG, Basel

Weining Xiong

Department of Respiratory Medicine

Shanghai Ninth People's Hospital, Shanghai Jiaotong University School of Medicine Zhizaoju Lu, Shanghai 200011 (China) xiongdoctor@qq.com

Yi Wang

Department of Respiratory and Critical Care Medicine, Tongji Hospital Tongji Medical College, Huazhong University of Science and Technology Jiefang Ave, Wuhan 430030 (China)

wangyi@tjh.tjmu.edu.cn 


\section{Introduction}

Asthma is a worldwide chronic lung disease characterized by recurrent bronchospasms, airway inflammation and hyperresponsiveness, mucus hypersecretion, and irreversible remodeling of the airways [1]. As a heterogeneous disease, both genetic and environmental factors are involved in the development of asthma. Therefore, it can be divided into several different phenotypes and subgroups that have varying therapeutic responses, especially following treatment with inhaled corticosteroids (ICS) $[2,3]$. Allergic asthma with the generation of allergen-specific $\mathrm{CD} 4^{+}$ $T$ cells as the initial event is the most common type of asthma. Naive T cells activated by antigen-presenting cells differentiate into T helper (Th) 2 cells $[4,5]$. Many studies dedicated that Th2 cells play a vital role in allergic asthma via interleukin (IL)-4, IL-5, IL-13 secretion which was associated with the production of allergen-specific IgE by B cells, airway inflammation and hyperresponsiveness, and inflammatory cell infiltration $[4,6]$. Virtually, allergic asthma is described as a Th2 inflammation of the airway $[6,7]$.

Epigenetics, such as DNA methylation, histone modification, and noncoding RNAs (ncRNAs) regulation, were involved in the pathogenesis of asthma [8,9]. Recent studies have shown that ncRNAs might play an important role in Th2-mediated inflammatory of asthma, likely to be attractive noninvasively biomarkers or targets for the treatment of asthma. For instance, the lncRNAsLNC_000127 functions in the Th2 inflammation pathway in eosinophilic asthma and targeting LNC_000127 may be effective for reducing Th2 inflammation [10]. The axis of miR-29c/B7-H3 participated in children with asthma through regulating Th2/Th17 cell differentiation and might provide new targets for the treatment of asthma [11]. Some studies also found that let-7e (previous ID of let-7e-5p) was downregulated in allergic rhinitis (AR) with asthma and emphasized the important role of let-7e in Th2-mediated allergic inflammation $[12,13]$.

CircRNAs as a new class of ncRNAs often function as molecular sponges to bind and inhibit microRNA (miRNA) transcription or activity, which subsequently affects the downstream mRNA expression [14]. Our previous study showed that circular RNA (circRNA) profiles were different and hsa_circ_0005519 regulated IL-13/IL-6 by sponging hsa-let-7a-5p in $\mathrm{CD}^{+} \mathrm{T}$ cells from asthma [15]. However, to our knowledge, the study of circRNAs in the regulation of asthma was in its infancy, and the contribution of circRNAs to the pathogenesis of asthma, especially to the Th2 inflammation of asthma, remains unknown. Here we hypothesized that circRNAs acting as endogenous hsa-let-7e-5p sponges might be involved in asthma, particularly in Th2-mediated allergic asthma and have potential diagnostic and therapeutic values. To verify our conjecture, we further analyzed our circRNA array and selected the one with the most significant difference for clinical validation in $\mathrm{CD} 4^{+} \mathrm{T}$ cells from asthmatic patients. We found that hsa_circ_0002594 in CD4 ${ }^{+} \mathrm{T}$ cells played a proinflammatory role in Th2-mediated allergic asthma. Moreover, hsa_circ_0002594 expression was downregulated by treatment with ICS. These observations suggested that hsa circ_0002594 was a proinflammatory factor in allergic asthma and that downregulation of hsa_circ_0002594 might partially account for the anti-inflammatory effect of ICS.

\section{Materials and Methods}

Subjects and Classification

156 subjects between 16 and 71 years in age were recruited from Tongji Hospital (Wuhan, China), including 102 asthmatic patients and 54 healthy subjects. None of the healthy subjects had a history of respiratory disease or evidence of bronchial hyperreactivity to methacholine challenge. All asthmatic patients had a doctor's diagnosis of symptomatic asthma and an accumulation dosage of methacholine provoking a $20 \%$ fall of forced expiratory volume in the first second $\left(\mathrm{FEV}_{1}, \mathrm{PD} 20\right)<2,505 \mathrm{mg}$ or bronchodilator responsiveness $\left(\geq 12 \%\right.$ improvement in $\mathrm{FEV}_{1} \%$ predicted and $\geq 200 \mathrm{~mL}$ improvement in $\mathrm{FEV}_{1}$ following inhalation of $200 \mu \mathrm{g}$ salbutamol) according to the Global Initiative for Asthma (GINA) guidelines. Among the 102 subjects with asthma, 83 had no course of any drug while 19 had a course of ICS at the first visit. The enrolled subjects were followed up for a second visit after treatment with ICS for 4 weeks. Written informed consent was obtained from all participants and all studies relevant to human subjects were approved by the Ethics Committee of Tongji Hospital, Huazhong University of Science and Technology. The composition of subjects included in the study was shown in online suppl. data 1 (for all online suppl. material, see www.karger.com/doi/10.1159/000511612).

\section{$C D 4^{+} \mathrm{T}$ Cells Isolation and RNA Extraction}

$\mathrm{CD}^{+} \mathrm{T}$ cells were isolated using density centrifugation and magnetic beads as described in our previous study [15]. Briefly, the diluted peripheral blood was carefully layered onto a FicollHypaque gradient to obtain peripheral blood mononuclear cells. Then, $\mathrm{CD}^{+} \mathrm{T}$ cells were isolated from peripheral blood mononuclear cells using a MagCellect Human CD $4^{+} \mathrm{T}$ Cell Isolation Kit (R\&D Systems, Minneapolis, MN, USA) according to the manufacturer's instructions. The isolated cells were frozen in TRIzol reagent (Takara, Dalian, China) at $-80^{\circ} \mathrm{C}$. The RNA purity and quantification were measured with a NanoDrop ND-2000 (Thermo, Waltham, MA, USA).

\section{Microarray and Quantitative Analysis}

$\mathrm{CD} 4^{+} \mathrm{T}$ cells of 5 asthmatic patients and 5 healthy subjects were homogenized in TRIzol reagent. The sample preparation and microarray hybridization were performed based on the Arraystar's standard protocols. The microarray and quantitative analysis were 
Table 1. Characteristics of subjects providing peripheral blood

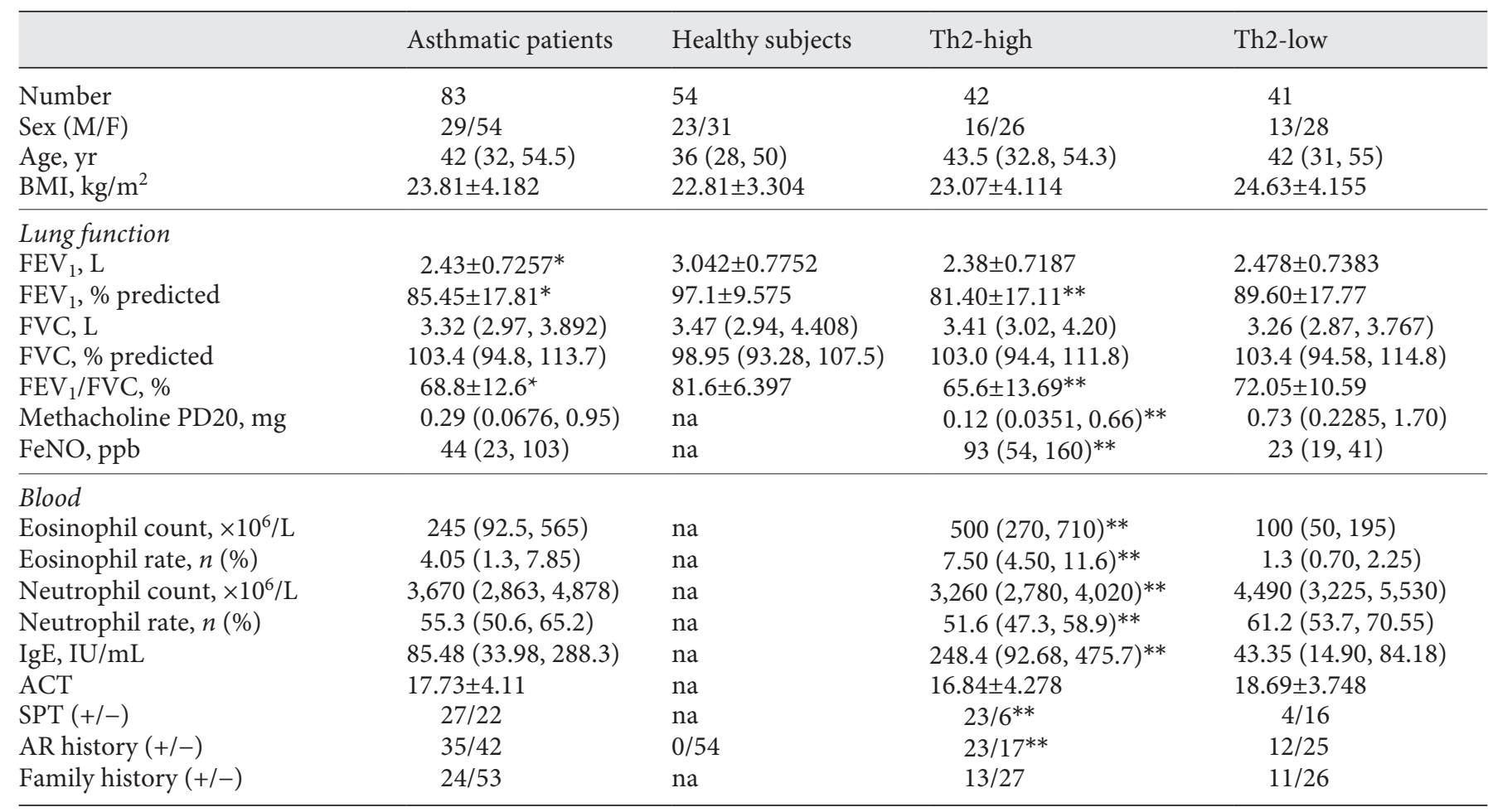

Blood counts were missing in 7 asthmatic patients. Serum IgE measurements were missing in 1 asthmatic patients. SPT was missing in 34 asthmatic patients; AR history and family history were unknown in 6 asthmatic patients. We expressed as the mean \pm SD and used paired or unpaired $t$ test for normally distributed data while we reported as medians with interquartile ranges and used nonparametric tests (Kruskal-Wallis test) for non-normally distributed data. Values are presented as mean $\pm \mathrm{SD}$ or median (interquartile range). FEV $\mathrm{V}_{1}$, forced expiratory volume in $1 \mathrm{~s}$; FVC, forced vital capacity; na, not applicable; PD20, provocation dose resulting in $20 \%$ fall in baseline $\mathrm{FEV}_{1}$; FENO, fraction exhaled nitric oxide; ACT, Asthma Control Test; SPT, skin prick test; AR, allergic rhinitis. ${ }^{*} p<0.05$ compared asthmatic patients with healthy subjects. ${ }^{* *} p<0.05$ compared Th2-high with Th2-low group.

described previously [15]. Quantile normalization and subsequent data processing were performed using the limma package [16]. The screening threshold was set to a fold change $>2.0$ or $<2.0$ with a value of $p<0.05$.

\section{Quantitative Reverse Transcription PCR ( $q R T-P C R$ )}

The RNA was reverse transcribed into cDNA using Prime Script RT MasterMix (Takara, Dalian, China) following the previous reports [15]. qRT-PCR was performed to quantify circRNA levels using an ABI Prism 7900 Real-Time System (Applied Biosystems, Foster City, CA, USA) with SYBR Premix Ex Taq (Takara, Dalian, China) following the manufacturer's instructions. The PCR parameters were the same as before [15]: $95^{\circ} \mathrm{C}$ for $30 \mathrm{~s}$, followed by 40 cycles of $95^{\circ} \mathrm{C}$ for $5 \mathrm{~s}, 60^{\circ} \mathrm{C}$ for $30 \mathrm{~s}$, and $72^{\circ} \mathrm{C}$ for $30 \mathrm{~s}$. The primers used in qRT-PCR are shown in online suppl. Table 2. $\beta$-Actin was used to normalize. The data were analyzed using the $2^{-\Delta \Delta \mathrm{Ct}}$ method and reported as the relative variation ( $\log _{2}$ transformed).

\section{Sanger Sequencing}

To verify the specificity of the divergent primers, we amplified the cDNA around the junction site of hsa_circ_0002594 in A549, followed by Sanger sequencing. The PCR was carried out for 35 cycles: denaturation at $95^{\circ} \mathrm{C}$ for $30 \mathrm{~s}$, annealing at $60^{\circ} \mathrm{C}$ for $30 \mathrm{~s}$, and elongation at $72^{\circ} \mathrm{C}$ for $30 \mathrm{~s}$. Sanger sequencing was performed by Geneseed Co. Ltd. (Guangzhou, China).

\section{ICS Treatment}

All subjects with asthma were placed on a standard ICS regimen (budesonide $200 \mu \mathrm{g}$ BID) by their personal physicians. Asthmatic patients with no ICS history continued to use ICS throughout 4 weeks period from the first visit to the second. A questionnaire was conducted to collect information and clinical data for the second visit. Characteristics of asthmatic patients with ICS were listed in online suppl. Table 3.

\section{Bioinformatics Analysis for hsa_circ_0002594}

MiRNA response elements (MREs) of hsa_circ_0002594 were predicted by Arraystar's miRNA target prediction software based on TargetScan and miRanda. The screened candidate targets of hsa_circ_0002594 were the integration of 3 online algorithms (miRanda, TargetScan, and PicTar or DIANA-microT-CDS). The circRNA/miRNA/mRNA interaction network was illustrated using Cytoscape software. 

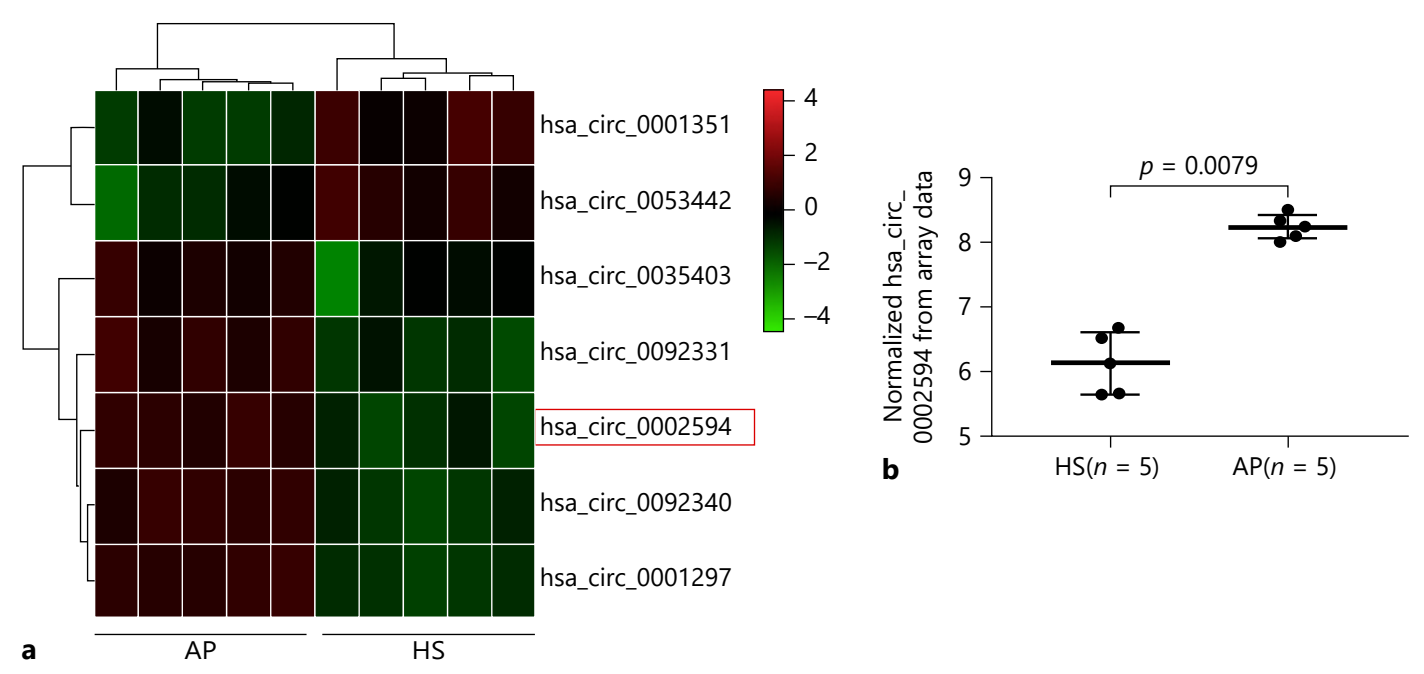

Fig. 1. Differential expression of circRNAs that could sponge hsalet-7e-5p in $\mathrm{CD}^{+} \mathrm{T}$ cells from asthmatic patients. a Hierarchical clustering analysis of differentially expressed circRNAs that could sponge hsa-let-7e-5p between AP and HS; each group included 5 subjects (fold change $>2 ; p<0.05$ ). Expression levels above and below the median expression level across all samples were represented in different colors. Pseudocolors indicate expression levels from low to high (green to red). b Hsa_circ_0002594 normalized expression levels were determined by the circRNA array in $\mathrm{CD}^{+}$ T cells from AP $(n=5)$ and HS $(n=5)$. c Schematic illustration of

\section{Statistical Analysis}

Statistical analyses were performed using SPSS 21.0 (IBM, SPSS, Chicago, IL, USA) and GraphPad Prism 7.0 (GraphPad, San Diego, CA, USA). We expressed as mean \pm SD and used paired or unpaired $t$ test for normally distributed data while we reported as medians with interquartile ranges and used nonparametric tests (Kruskal-Wallis test) for non-normally distributed data. Fisher's exact test was performed on analysis of categorical data and Spearman's rank-order correlation for correlation analysis. Receiver operating characteristic (ROC) was generated to determine the diagnostic value of hsa_circ_0002594. Differences were considered significant at a $p$ value of $<0.05$.

Hsa_circ_0002594 in Th2-Mediated Allergic Asthma the genomic location and splicing pattern of hsa_circ_0002594, which formed by head-to-tail splicing of 2 exons. Sanger sequencing depicted the splice junction. d The expression of hsa circ_0002594 was analyzed in CD4 ${ }^{+} \mathrm{T}$ cells ( $n=83$ for AP; $n=54$ for HS). Values were relative to the median value of healthy subjects and expressed as $\log _{2}$ transformed, normalized to $\beta$-actin. The values presented were the mean \pm SD. $p$ values were calculated by Kruskal-Wallis test. circRNAs, circular RNAs; HS, healthy subjects; AP, asthmatic patients without ICS.

\section{Results}

Overview of Asthmatic Patients without ICS and Healthy Subjects

A questionnaire was conducted to collect information and clinical data for 83 patients with asthma and 54 healthy subjects. Participants were excluded if they had a respiratory tract infection in the previous 4 weeks. Clinical data for asthmatic patients without ICS were shown in Table 1. There were no significant differences across asthmatic pa- 


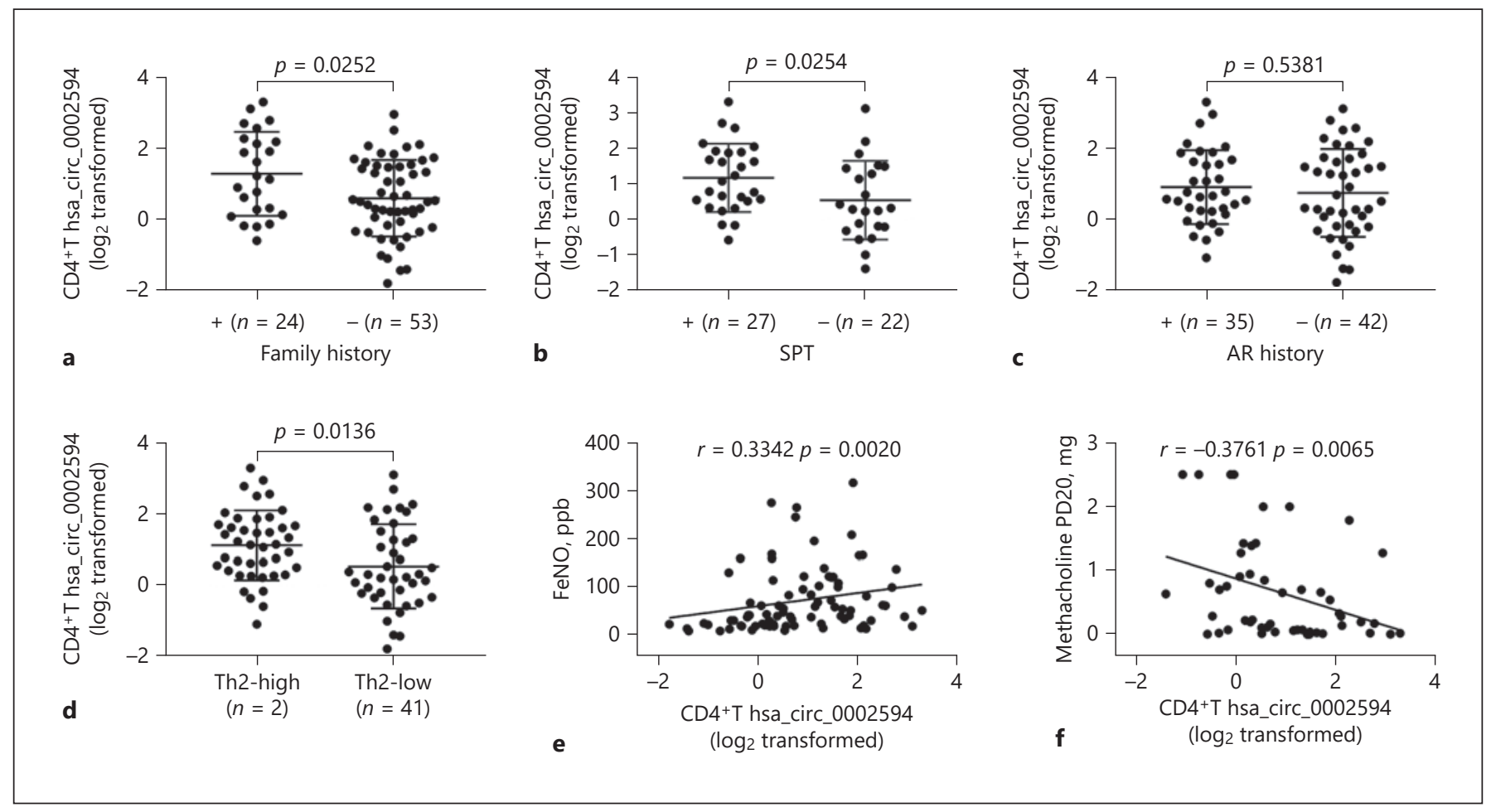

Fig. 2. Hsa_circ_0002594 expression in different subgroups and its contribution to Th2 immune activation of asthma. Hsa circ_0002594 expressions in patients with or without a family history (a), patients with a positive or negative SPT (b), patients with or without AR (c), and patients with Th2-high or Th2-low (d). e Correlation assay between hsa_circ_0002594 levels and FeNO in asthmatic patients $(n=83)$. $\mathbf{f}$ Correlation assay between hsa circ_0002594 levels and PD20 in asthmatic patients $(n=51)$. We used nonparametric tests (Kruskal-Wallis test) for non-normally distributed data. Fisher's exact test was performed on analysis of categorical data and Spearman's rank-order correlation for correlation analysis. SPT, skin pricking test; AR, allergic rhinitis; Th, T helper. tients and healthy subjects in terms of sex distributions, age, and BMI. Eighty-three subjects were classified as Th2-high or Th2-low asthma based on biomarkers reflecting Th2 immune activation, including blood eosinophil count, IgE level, and fraction of exhaled nitric oxide (FeNO) concentration. Th2-high indicated an elevation in 2 or more Th2 biomarkers while Th2-low indicated an elevation in 1 or no Th2 biomarker. For each biomarker, cut points used to define a high level were as follows: eosinophil count $\geq 300 / \mu \mathrm{L}$ or eosinophil rate $\geq 3 \%$, IgE $\geq 100 \mathrm{IU} /$ $\mathrm{mL}$, and $\mathrm{FeNO} \geq 50$ parts per billion $[17,18]$.

\section{Expression of hsa_circ_0002594 in CD4 $4^{+}$T Cells of Asthmatic Patients}

To identify the roles of circRNAs that could act as an endogenous hsa-let-7e-5p sponge in the pathogenesis of asthma, we found 7 differentially expressed circRNAs in our circRNA array of $\mathrm{CD}^{+} \mathrm{T}$ cell samples from 5 asthmatic patients and 5 healthy controls as mentioned in our previous study [15]. The 5 up- and 2 downregulated circRNAs were listed in online suppl. 4. The expression profiles of 7 selected circRNAs were shown by hierarchical clustering in Figure 1a, and our array data revealed that hsa_circ_0002594 (fold change $\geq 4.3$ ) was one of the most upregulated circRNAs in $\mathrm{CD} 4^{+} \mathrm{T}$ cells of asthmatic patients (Fig. 1b). The cDNA around the junction site of hsa_circ_0002594 was detected by Sanger sequencing and the results were consistent with the circBase data (Fig. 1c). Another cohort study including 83 asthmatic patients and 54 healthy subjects further verified the results of the circRNA array that hsa_circ_0002594 levels were markedly increased in $\mathrm{CD}^{+} \mathrm{T}$ cells from asthmatics (Fig. 1d).

\section{Hsa_circ_0002594 Contributed to Clinical}

Characteristics Especially Th2 Immune Activation of

Asthma

We further analyzed the correlations between hsa circ_0002594 expression and various clinical parameters 


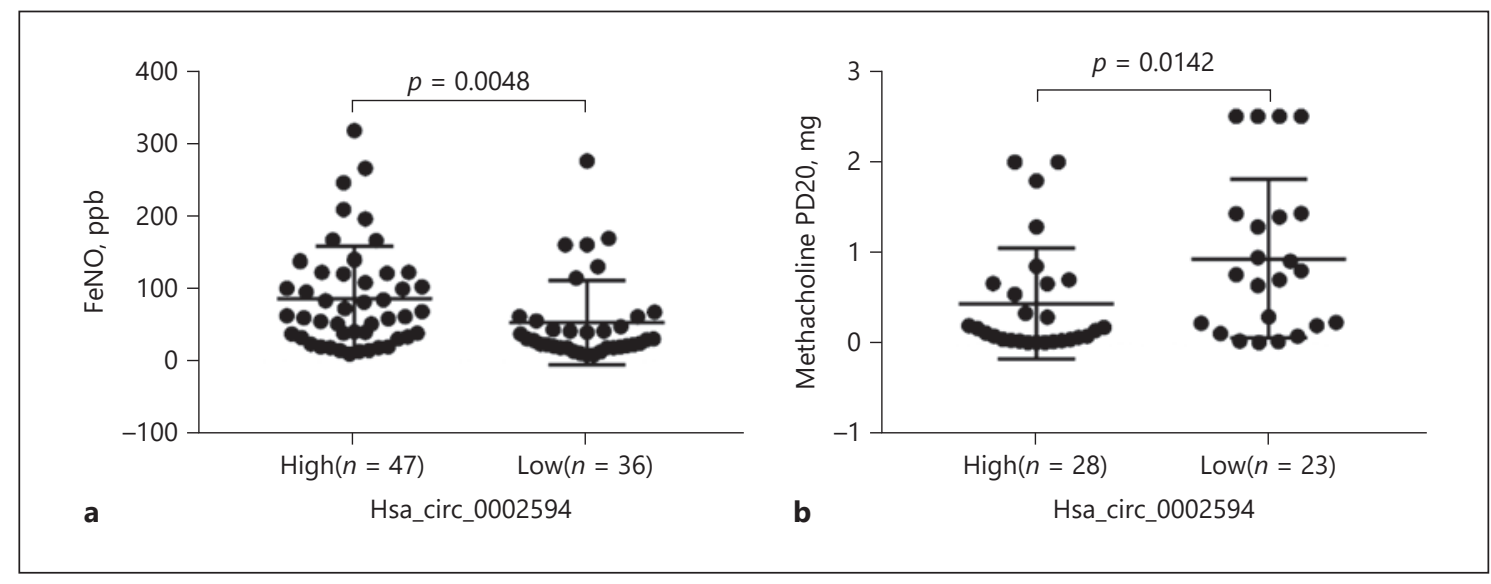

Fig. 3. Hsa_circ_0002594-high asthma was characterized by clinical indicators of allergic asthma. Hsa_ circ_0002594-high subgroup seemed to be characterized by a higher FeNO (a) and a lower PD20 (b). p values were calculated by Kruskal-Wallis test.

Table 2. Th2-high and Th2-low and SPT frequencies in asthmatic patients with high or low expressions of hsa_circ_0002594

\begin{tabular}{lcccc}
\hline & \multicolumn{2}{l}{ Hsa_circ_0002594 } & OR (95\% CI) & $\begin{array}{l}p \\
\text { value }\end{array}$ \\
\cline { 2 - 3 } & high & low & & \\
\hline Th2 & & & & \\
High & 30 & 12 & $3.5(1.42-8.80)$ & 0.0079 \\
Low & 17 & 24 & & \\
\hline SPT & & & & \\
Positive & 20 & 9 & $3.6(1.09-11.86)$ & 0.0234 \\
Negative & 7 & 13 & & \\
\hline
\end{tabular}

See Table 1 legend for expansion of abbreviations. $p<0.05$ was considered statistically significant. Fisher's exact test was performed on analysis of categorical data. OR, odds ratio; CI, confidence interval; Th, T helper; SPT, skin prick test.

of asthma. In 83 asthmatic patients without ICS, hsa circ_0002594 expression was found higher in patients with a family history or a skin pricking test (SPT)-positive group than those with no family history or a SPT-negative group (Fig. 2a, b). Allergic asthma was a Th2-dominated disease, with Th2 cells being prevalent in AR, a common comorbidity with asthma $[13,19]$. However, hsa circ_0002594 levels were comparable in the asthmatics with or without AR (Fig. 2c). Interestingly, when we divided asthmatic patients into Th2-high and Th2-low subgroups, Th2-high revealed higher expression of hsa circ_0002594 (Fig. 2d). There was also a positive correlation between hsa_circ_0002594 levels and FeNO, a parameter of Th2 immune activation and a good predic-

Hsa_circ_0002594 in Th2-Mediated

Allergic Asthma tor of corticosteroid response [17, 20] (Fig. 2e). We also observed that the expression of hsa_circ_0002594 was negatively correlated with methacholine PD20 in 51 asthmatic patients (Fig. 2f). Collectively, these findings suggested that elevated hsa_circ_0002594 levels might be correlated with Th2-mediated allergic asthma.

\section{Hsa_circ_0002594-High Asthma Is Characterized by} Multiple Clinical Indicators of Th2-Mediated Allergic Asthma

When we used the lower limit of the upper quartile of hsa_circ_0002594 expression level ( $\log _{2}$ transformed $=$ 0.53143125 ) of the healthy controls as the cutoff point, theasthmaticpatientsweredividedintohsa_circ_0002594low and hsa_circ_0002594-high subgroups. Compared with the hsa_circ_0002594-low group, the hsa circ_0002594-high group had higher FeNO and lower PD20 (Fig. 3a, b). Moreover, in the hsa_circ_0002594high subgroup, the frequencies of Th2-high and SPTpositive were both higher, 3.5 and 3.6 times more likely than non-Th2 inflammation and SPT-negative, respectively (Table 2). Correlations between hsa_circ_0002594high asthma and clinical characteristics were listed in Table 3. To sum up, hsa_circ_0002594-high asthma was characterized by multiple clinical indicators of Th2-mediated allergic asthma.

\section{Diagnostic Value and Therapeutic Response of hsa_circ_0002594}

To identify the diagnostic value of hsa_circ_0002594, 83 asthmatic patients without ICS and 54 healthy subjects were analyzed to construct a ROC curve, with an area un- 
Table 3. Comparison of patients in the clinical trials used to define hsa_circ_0002594-high and hsa_circ_0002594-low subgroups

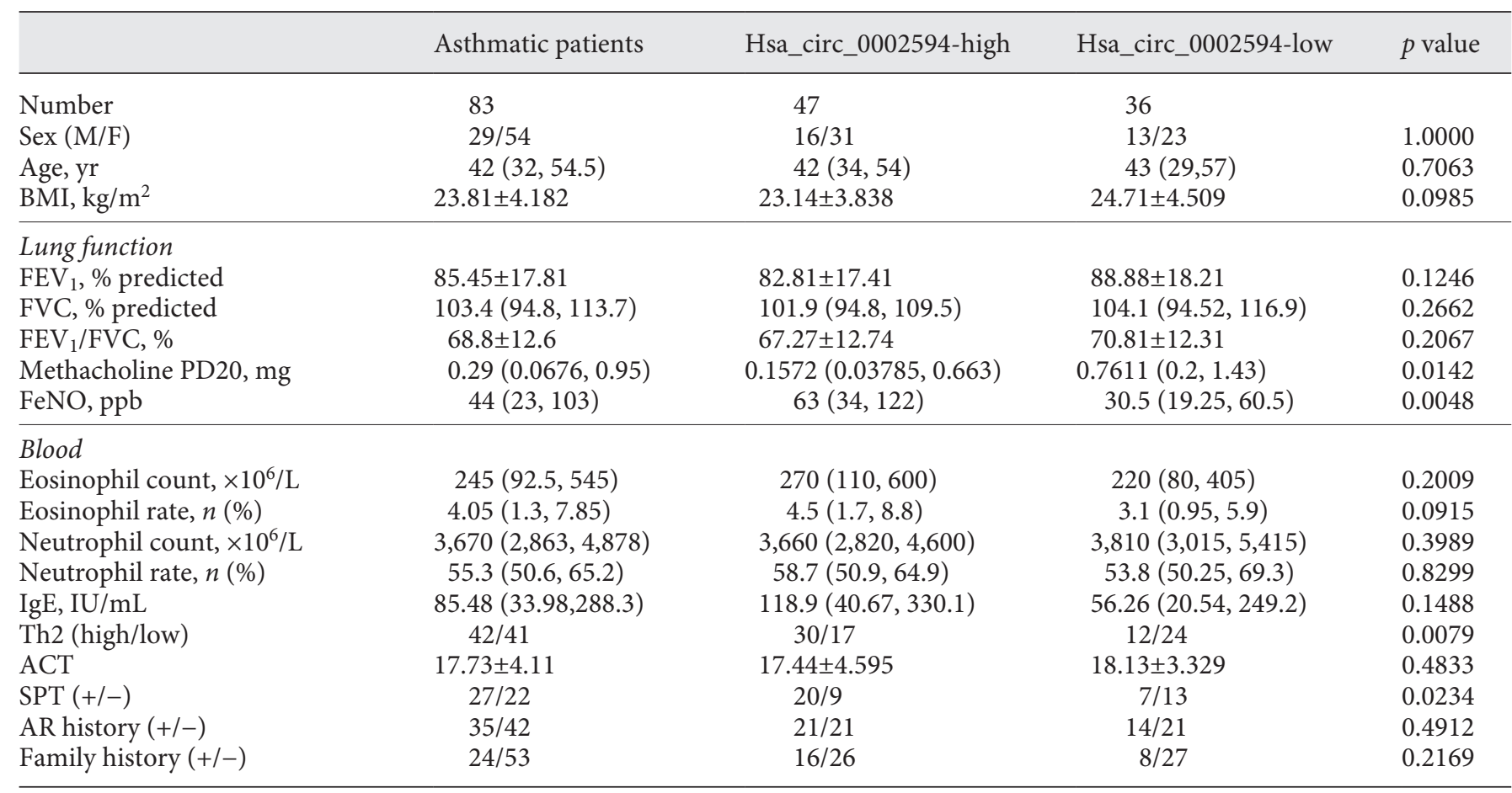

See Table 1 legend for expansion of abbreviations. $p$ for comparison between hsa_circ_0002594-high and hsa_circ_0002594-low subgroups. $p<0.05$ was considered statistically significant. $p$ values were calculated by unpaired 2-sided Student's $t$ test, Kruskal-Wallis test, or Fisher's exact test, as appropriate. Blood counts were missing in 4 hsa_circ_0002594-high and 3 hsa_circ_0002594-low subgroups. Serum IgE measurements were missing in 1 hsa_circ_0002594-low subgroup. SPT was missing in 19 hsa_circ_0002594-high and 15 hsa_circ_0002594-low subgroups. AR history and family history were unknown in 5 hsa_circ_0002594-high and 1 hsa_circ_0002594low subgroups. $\mathrm{FEV}_{1}$, FVC, forced vital capacity; PD20, provocation dose resulting in $20 \%$ fall in baseline FEV $\mathrm{FENO}_{1}$, fraction exhaled nitric oxide; Th, T helper; ACT, Asthma Control Test; SPT, skin prick test; AR, allergic rhinitis.

der the curve (AUC) value of $0.7278(p<0.0001$, Fig. 4a). To explore the therapeutic response of hsa_circ_0002594, the levels of hsa_circ_0002594 in CD4 ${ }^{+}$T cells originated from subjects with asthma after 4 weeks of ICS treatment were determined. Interestingly, hsa_circ_0002594 levels were obviously decreased when compared with their first visit ( $n=29$, Fig. $4 \mathrm{~b})$. The same results were observed in asthmatic subjects with ICS $(n=48)$ including another 19 subjects with a course of ICS when compared with 83 subjects without ICS at their first visit (Fig. 4c). When examining the diagnostic value of hsa_circ_0002594 in the presence of ICS, an AUC value of $0.7671(p<0.0001$, Fig. 4d) was obtained.

\section{Construction of circRNA-miRNAs-mRNAs Network}

Importantly, circRNAs have been demonstrated to act as miRNA sponges to regulate gene expression. They could naturally function as competing endogenous RNA by competing with mRNAs for the same MREs [14,21]. To further explore the functions of hsa_circ_0002594, we constructed the hsa_circ_0002594-miRNAs-mRNAs network based on bioinformatics and the mRNA profile published by our group previously [22]. First, the potential MREs of hsa circ_0002594 predicted by Arraystar's miRNA target prediction software were hsa-let-7e-5p, hsa-miR-16-5p, hsamiR-503-5p, hsa-miR-514a-3p, and hsa-miR-587 (Fig. 5a). Then, 222 screened candidate targets of miRNAs were the merged common targets of 3 online algorithms and mRNA profile (online suppl. 5). Finally, the entire network was illustrated using Cytoscape software (Fig. 5b).

\section{Discussion}

CircRNAs acting as miRNA sponges played a critical role in the occurrence and development of many diseases associated with abnormal immune response including systemic lupus erythematous, rheumatoid arthritis, 


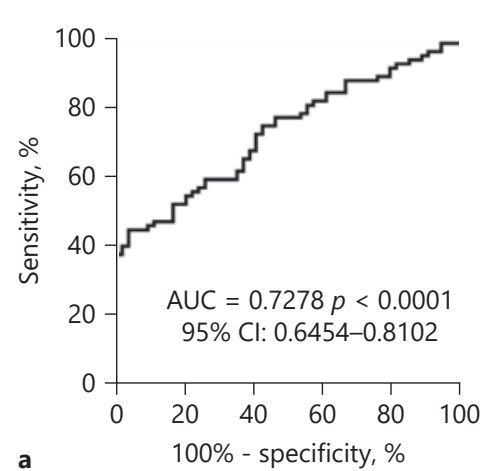

a

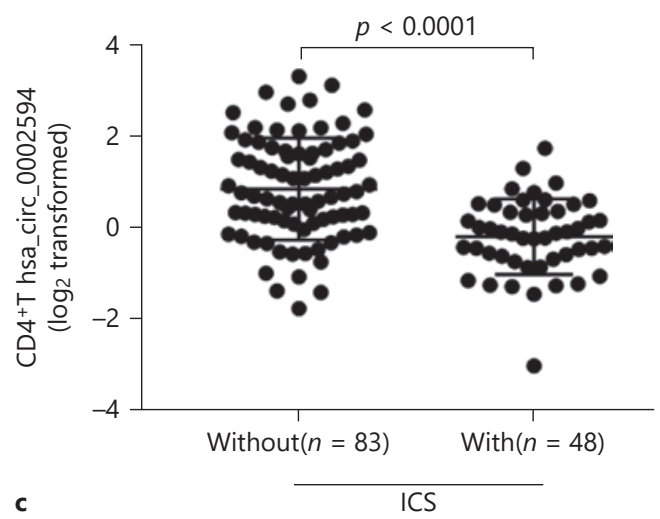

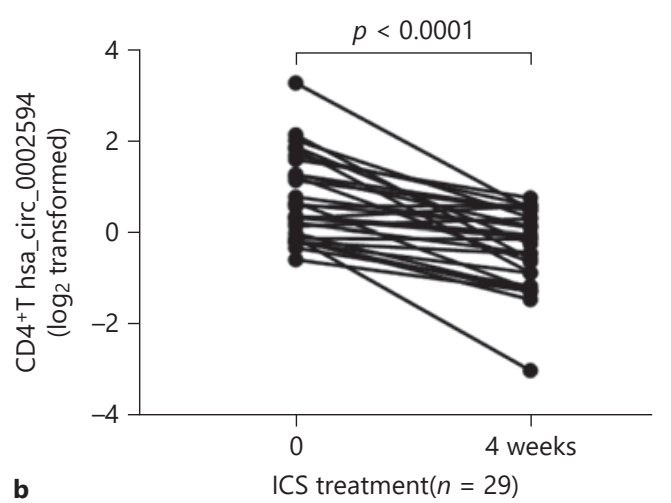

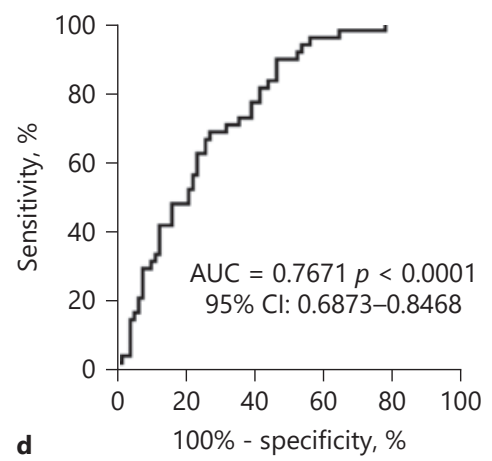

Fig. 4. Diagnostic and therapeutic value of hsa_circ_0002594 for Th2-mediated allergic asthma. a ROC curve analyses of hsa_circ_0002594 in 83 asthmatic patients without ICS. b Hsa_circ_0002594 levels were decreased after 4 weeks of ICS treatment $(n=29)$. $p$ values were calculated by paired $t$ test. c Hsa_circ_0002594 levels were lower in asthmatic subjects with ICS $(n=48)$. $p$ values were calculated by Kruskal-Wallis test. d ROC curve analyses of hsa_circ_0002594 in the presence of ICS $(n=48)$. ROC, receiver operating characteristic; AUC, area under the curve; ICS, inhaled corticosteroids.

Crohn disease, and diabetes [23-26]. In our previous study, we demonstrated circRNAs profiles and hsa circ_0005519 regulated IL-13/IL-6 by sponging hsa-let$7 a-5 p$ in $\mathrm{CD}^{+} \mathrm{T}$ cells from asthma [15]. But the expression profiles and functions of circRNAs in asthma were still scarce. In the present study, we found 7 differentially expressed circRNAs that might act as an endogenous hsalet-7e-5p sponge in our circRNA array [15], among which hsa_circ_0002594 was upregulated in another cohort $\mathrm{CD}^{+} \mathrm{T}$ cells of allergic asthma for further verification.

Current studies have found that the pathogenesis of asthma was closely related to the imbalance of T cells especially the helper T cells subgroups (Th1, Th2, Th9, Th17, Treg, etc.), and Th2 played a crucial role in asthma allergic airway inflammation [27-31]. In previous study, Guan et al. [32] demonstrated that let-7e played a critical role in the differentiation of Th1, Th2, and Th17 cells and negatively regulated Th2 polarization as well as IL-10 production. In addition, let-7e was downregulated in AR with asthma and emphasized the important role of let-7e in Th2-mediated allergic inflammation $[12,13]$. Given the roles of circRNAs as miRNA "sponges" to regulate gene expression [14], we conceived that some circRNAs could sponge let-7e to participate in Th2-mediated allergic asthma.

In this study, we found 7 differentially expressed circRNAs that could act as an endogenous hsa-let-7e-5p in our circRNA array, and hsa_circ_0002594 that exhibited the most significant change in the 7 circRNAs was selected for follow-up study. Subsequent qRT囚PCR validation suggested that hsa_circ_0002594 was upregulated in $\mathrm{CD}^{+} \mathrm{T}$ cells of asthma, matching with the microarray results. We also demonstrated that hsa_circ_0002594 ex- 


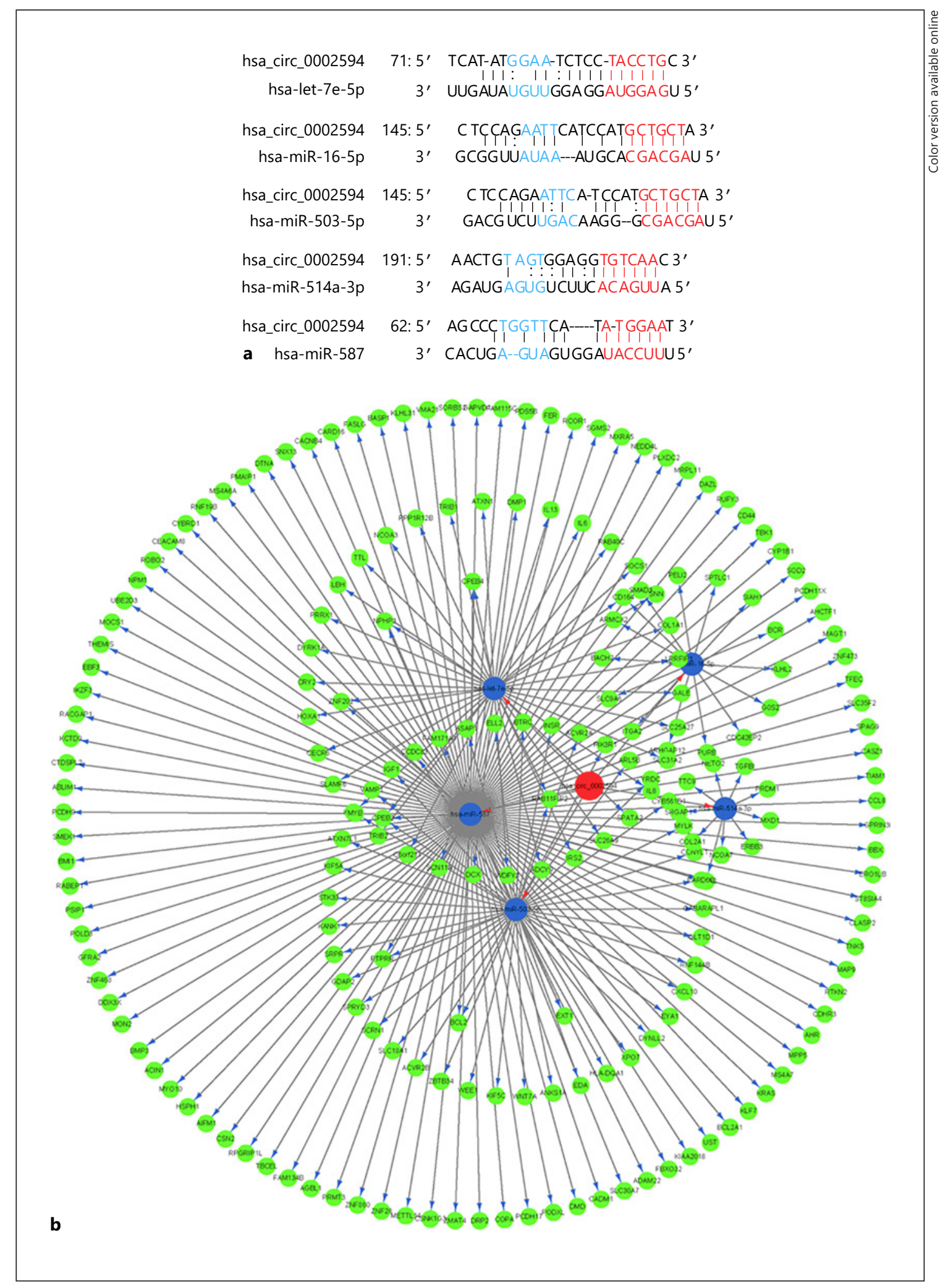

Fig. 5. Bioinformatics analysis of hsa_circ_0002594. a Detailed annotation for the hsa_circ_0002594/miRNAs interaction. b The hsa_circ_0002594-miRNAs-mRNAs network. Red nodes: hsa_circ_0002594; blue nodes: miRNAs; green nodes: mRNAs. miRNAs, microRNA. 
pression was higher in subjects with a family history, SPT-positive, or Th2-high. Hsa_circ_0002594 levels were positively correlated with FeNO and negatively correlated with methacholine PD20. Hsa_circ_0002594-high asthma was characterized by multiple clinical indicators of allergic asthma such as FeNO, Th2-high phenotype, PD20, etc. These results indicated that hsa_circ_0002594 might be involved in Th2-mediated allergic asthma. Furthermore, hsa_circ_0002594 was decreased after ICS treatment. Moreover, hsa_circ_0002594 showed high ROC AUC values with or without ICS respectively, indicating its high potential diagnostic and therapeutic value for asthma. In conclusion, hsa_circ_0002594 might act as a promising diagnostic or therapeutic biomarker for Th2mediated allergic asthma. In addition, to further explore the possible molecular mechanism of hsa_circ_0002594 in asthma, we identified that hsa_circ_0002594 might target hsa-let-7e-5p, hsa-miR-16-5p, hsa-miR-503-5p, hsamiR-514a-3p, and hsa-miR-587 based on bioinformatics and thus competitively sequester miRNA activity.

Our study had some limitations. First, considering the limitations of our sample size, more samples from different regions were needed to confirm our results. Second, we hypothesized the possible mechanism by which hsa circ_0002594 was involved in allergic asthma mainly based on bioinformatics analysis, but no in vitro studies were conducted. Third, circulating hsa_circ_0002594 was not detected to assess its potential as a noninvasive biomarker for the diagnosis and therapy of allergic asthma.

In conclusion, hsa_circ_0002594 was upregulated in $\mathrm{CD}^{+}{ }^{+} \mathrm{T}$ cells of allergic asthma. Our findings might enhance the knowledge regarding the role of circRNAs in asthma since there were few studies in this field. In addition, hsa_circ_0002594 might have value in the diagnosis and treatment of Th2-mediated allergic asthma. These observations should have potential clinical significance, and might provide new insight into the molecular mechanisms of allergic asthma, all of which merit further investigation.

\section{Acknowledgements}

We were grateful to all the study investigators, staff, and patients who participated in this study for helpful discussions during manuscript development.

\section{Statement of Ethics}

The Ethics Committee of Tongji Hospital, Tongji Medical College, Huazhong University of Science and Technology approved this study (IRB ID: 20150503). All procedures performed in the study involving human participants were in accordance with the ethical standards of the institutional research committee.

\section{Conflict of Interest Statement}

The authors have no conflicts of interest to declare.

\section{Funding Sources}

This work was supported by grants from National Natural Science Foundation of China (No. 91742111, No. 81570024), Wuhan Young and Middle-aged Medical Key Talents Training Project, Clinical Research Physician Program of Tongji Medical College, HUST, and Integrated Innovative Team for Major Human Diseases Program of Tongji Medical College, HUST.

\section{Author Contributions}

H.Z. recruited patients; collected, analyzed, and interpreted the data; and wrote the draft. F.B., Q.X., X.Y., M.Y., and W.G. recruited patients and collected data. W.Y., Z.M., and C.Y. analyzed and interpreted the data, and critically revised the manuscript. W.Y., Z.J., and X.W. conceived and designed the study, recruited patients, and drafted the manuscript for important intellectual content. X.W., X.J., and Z.J. provided overall supervision and critically revised the manuscript. All authors read and approved the final manuscript.

\section{References}

1 Wang Y, Zhu J, Zhang L, Zhang Z, He L, Mou $\mathrm{Y}$, et al. Role of C/EBP homologous protein and endoplasmic reticulum stress in asthma exacerbation by regulating the IL-4/signal transducer and activator of transcription 6/ transcription factor EC/IL-4 receptor a positive feedback loop in M2 macrophages. J Allergy Clin Immunol. 2017;140(6):1550-61 e8.

2 Wenzel SE. Asthma phenotypes: the evolution from clinical to molecular approaches. Nat Med. 2012;18(5):716-25.
3 Lei Z, Yanhan D, Yi W, Shengdao X, Weining $\mathrm{X}$. Perimenstrual asthma: report of a case with therapeutic intervention of oral prednisone. J Asthma. 2018;55(12):1328-30.

4 Romagnani S. Immunologic influences on allergy and the TH1/TH2 balance. J Allergy Clin Immunol. 2004;113(3):395-400.

5 Craig TJ. Aeroallergen sensitization in asthma: prevalence and correlation with severity. Allergy Asthma Proc. 2010;31(2):96-102.

6 Lambrecht BN, Hammad H. The immunology of asthma. Nat Immunol. 2015;16(1):45-56.
7 Kim HY, DeKruyff RH, Umetsu DT. The many paths to asthma: phenotype shaped by innate and adaptive immunity. Nat Immunol. 2010;11(7):577-84.

8 Ho SM. Environmental epigenetics of asthma: an update. J Allergy Clin Immunol. 2010; 126(3):453-65.

9 Alizadeh Z, Mortaz E, Adcock I, Moin M. Role of epigenetics in the pathogenesis of asthma. Iran J Allergy Asthma Immunol. 2017;16(2):82-91.
Hsa_circ_0002594 in Th2-Mediated Allergic Asthma
Int Arch Allergy Immunol 2021;182:388-398 DOI: $10.1159 / 000511612$ 
10 Zhu Y, Mao D, Gao W, Han G, Hu H. Analysis of lncRNA expression in patients with eosinophilic and neutrophilic asthma focusing on LNC 000127. Front Genet. 2019;10:141.

11 Zhang X, Zhao X, Sun H, Yan Y, Huang L, Gu $\mathrm{W}$, et al. The role of miR-29c/B7-H3 axis in children with allergic asthma. J Transl Med. 2018;16(1):218.

12 Suojalehto $\mathrm{H}$, Toskala E, Kilpeläinen $\mathrm{M}, \mathrm{Ma}$ juri ML, Mitts C, Lindström I, et al. MicroRNA profiles in nasal mucosa of patients with allergic and nonallergic rhinitis and asthma. Int Forum Allergy Rhinol. 2013;3(8):612-20.

13 Suojalehto H, Lindström I, Majuri ML, Mitts C, Karjalainen J, Wolff $\mathrm{H}$, et al. Altered microRNA expression of nasal mucosa in longterm asthma and allergic rhinitis. Int Arch Allergy Immunol. 2014;163(3):168-78.

14 Hansen TB, Jensen TI, Clausen BH, Bramsen JB, Finsen B, Damgaard CK, et al. Natural RNA circles function as efficient microRNA sponges. Nature. 2013;495(7441):384-8.

15 Huang Z, Cao Y, Zhou M, Qi X, Fu B, Mou Y, et al. Hsa_circ_0005519 increases IL-13/IL-6 by regulating hsa-let-7a-5p in CD4. Clin Exp Allergy. 2019;49(8):1116-27.

16 Ritchie ME, Phipson B, Wu D, Hu Y, Law $\mathrm{CW}$, Shi W, et al. limma powers differential expression analyses for RNA-sequencing and microarray studies. Nucleic Acids Res. 2015; 43(7):e47.

17 Busse WW, Holgate ST, Wenzel SW, Klekotka P, Chon Y, Feng J, et al. Biomarker profiles in asthma with high vs. low airway reversibility and poor disease control. Chest. 2015; 148(6):1489-96.
18 Dweik RA, Boggs PB, Erzurum SC, Irvin CG, Leigh MW, Lundberg JO, et al. An official ATS clinical practice guideline: interpretation of exhaled nitric oxide levels (FENO) for clinical applications. Am J Respir Crit Care Med. 2011;184(5):602-15.

19 Cruz AA, Popov T, Pawankar R, Annesi-Maesano I, Fokkens W, Kemp J, et al. Common characteristics of upper and lower airways in rhinitis and asthma: ARIA update, in collaboration with GA(2)LEN. Allergy. 2007;62(Suppl 84):1-41.

20 Silkoff PE, Lent AM, Busacker AA, Katial RK, Balzar S, Strand M, et al. Exhaled nitric oxide identifies the persistent eosinophilic phenotype in severe refractory asthma. J Allergy Clin Immunol. 2005;116(6):1249-55.

21 Greene J, Baird AM, Brady L, Lim M, Gray SG, McDermott R, et al. Circular RNAs: biogenesis, function and role in human diseases. Front Mol Biosci. 2017;4:38

22 Qi X, Chen H, Huang Z, Fu B, Wang Y, Xie J, et al. Aberrantly expressed lncRNAs identified by microarray analysis in CD4+ T cells in asthmatic patients. Biochem Biophys Res Commun. 2018;503(3):1557-62.

23 Ouyang Q, Wu J, Jiang Z, Zhao J, Wang R, Lou A, et al. Microarray expression profile of circular RNAs in peripheral blood mononuclear cells from rheumatoid arthritis patients. Cell Physiol Biochem. 2017;42(2):651-9.

24 Zhao Z, Li X, Jian D, Hao P, Rao L, Li M. Hsa circ_0054633 in peripheral blood can be used as a diagnostic biomarker of pre-diabetes and type 2 diabetes mellitus. Acta Diabetol. 2017; 54(3):237-45.
25 Zhang C, Wang X, Chen Y, Wu Z, Zhang C, Shi W. The down-regulation of hsa_circ_0012919, the sponge for miR-125a-3p, contributes to DNA methylation of CD11a and CD70 in CD4+ T cells of systemic lupus erythematous. Clin Sci. 2018;132(21):2285-98.

26 Yin J, Hu T, Xu L, Li P, Li M, Ye Y, et al. Circular RNA expression profile in peripheral blood mononuclear cells from Crohn disease patients. Medicine. 2019;98(26):e16072.

27 Looman KIM, van Meel ER, GrosserichterWagener C, Vissers FJM, Klingenberg JH, de Jong NW, et al. Associations of Th2, Th17, Treg cells, and IgA+ memory B cells with atopic disease in children: the generation $\mathrm{R}$ Study. Allergy. 2019;18(4):765-76.

28 Choy DF, Hart KM, Borthwick LA, Shikotra A, Nagarkar DR, Siddiqui S, et al. TH2 and TH17 inflammatory pathways are reciprocally regulated in asthma. Sci Transl Med. 2015; 7(301):301ra129.

29 Tumes DJ, Papadopoulos M, Endo Y, Onodera A, Hirahara K, Nakayama T. Epigenetic regulation of T-helper cell differentiation, memory, and plasticity in allergic asthma. Immunol Rev. 2017;278(1):8-19.

30 Angkasekwinai P. Th9 cells in allergic disease. Curr Allergy Asthma Rep. 2019;19(5):29.

31 Leomicronn B. T cells in allergic asthma: key players beyond the Th2 pathway. Curr Allergy Asthma Rep. 2017;17(7):43.

32 Guan H, Fan D, Mrelashvili D, Hao H, Singh NP, Singh UP, et al. MicroRNA let-7e is associated with the pathogenesis of experimental autoimmune encephalomyelitis. Eur J Immunol. 2013;43(1):104-14. 